Cahiers $d u$ MONDE RUSSE

\section{Cahiers du monde russe}

Russie - Empire russe - Union soviétique et États indépendants

$51 / 1 \mid 2010$

Pierre le Grand et ses images de Rome

\title{
La référence romaine dans la bibliothèque de Pierre le Grand
}

\section{Olga Medvedkova}

\section{(Q) OpenEdition \\ Journals}

Édition électronique

URL : https://journals.openedition.org/monderusse/9171

DOI : 10.4000/monderusse. 9171

ISSN : $1777-5388$

Éditeur

Éditions de l'EHESS

\section{Édition imprimée}

Date de publication : 15 avril 2010

Pagination : 65-70

ISBN : 978-2-7132-2306-8

ISSN : $1252-6576$

Référence électronique

Olga Medvedkova, "La référence romaine dans la bibliothèque de Pierre le Grand ", Cahiers du monde russe [En ligne], 51/1 | 2010, mis en ligne le 10 mai 2013, consulté le 03 septembre 2022. URL : http:// journals.openedition.org/monderusse/9171 ; DOI : https://doi.org/10.4000/monderusse.9171

Ce document a été généré automatiquement le 3 septembre 2022 


\title{
La référence romaine dans la bibliothèque de Pierre le Grand
}

\author{
Olga Medvedkova
}

1 Depuis sa création et tout au long de son histoire, la ville de Saint-Pétersbourg fut toujours marquée par le modèle romain. Ceci n'a rien d'exceptionnel. Dans l'Europe de l'époque moderne, la création des villes nouvelles ayant pour but la reproduction de l'idée même de la centralité - universelle, nationale, régionale etc. - s'accompagnait régulièrement d'une référence à la Rome, aussi bien celle des empereurs que celle des papes.

2 Sur ce fonds, le «cas » pétersbourgeois est, à la fois, d'une évidence extrême - à commencer par le nom de la ville et ses armes et à terminer par ses immenses places en forme de cirques parsemés d'arcs et de colonnes de triomphe-, et d'un flou tout aussi remarquable. En effet, dès qu'il s'agit d'expliquer cette évidence, nous sommes confrontés à une pénurie de sources nous empêchant de mettre en place tout outil d'interprétation satisfaisante.

3 Ainsi, depuis le célèbre essai de Jurij Lotman et Boris Uspenskij ${ }^{1}$, qui ont développé - de manière aussi brillante que, parfois, anachronique - l'idée de Saint-Pétersbourg, troisième Rome, nous n'observons pas d'évolution importante dans le traitement d'un sujet aussi primordial pour l'histoire de la culture russe. Une source telle que la bibliothèque de Pierre le Grand qui, en partie du moins, est conservée encore aujourd'hui dans les réserves de la bibliothèque de l'Académie des sciences (BAN) de SaintPétersbourg, a été notamment négligée jusqu'à présent. Or, cette bibliothèque contenant les ouvrages que le prince fondateur avait sous les yeux au moment de la création de sa nouvelle ville devenue capitale permet de reposer la question de sa conception, aussi bien spatiale que symbolique.

4 Cette situation s'explique par l'introduction généralement lente des bibliothèques dans le cadre des études historico-culturelles et, en particulier, par la connaissance insuffisante de la bibliothèque en question, cataloguée de manière succincte et défectueuse dans les années $1970^{2}$. Elle s'explique, aussi, par les problèmes que pose la nature cosmopolite de la bibliothèque de Pierre le Grand, qui ne peut être 
authentiquement comprise que si l'on prend en compte tous ses éléments constitutifs. Or, une telle vision, à la fois globale et détaillée, nécessite un concert de compétences hors du commun.

5 Le projet d'étude de la bibliothèque de Pierre le Grand, que nous avons lancé en 2005 grâce au soutien de l'Observatoire français en sciences sociales de Moscou, de la Maison des sciences de l'homme et du Centre d'études des mondes russe, caucasien et centreeuropéen de l'École des hautes études en sciences sociales, a permis de réunir les conditions préliminaires à la réintroduction de cette source exceptionnelle dans la circulation scientifique. La rencontre entre les équipes d'historiens européens (depuis les premières réflexions et jusqu'à aujourd'hui ont participé à ce projet Wladimir Berelowitch, Pascal Dubourg-Glatigny, Peter Fuhring, Daniela Gallo, Claude Mignot, Émilie d'Orgeix, Philippe Sénéchal, Dirk Van der Vijver) et russes (Irina Beljaeva, Irina Hmelevskih, Inga Lander, Irina Lebedeva, Elena Savel'eva) a permis de lancer le travail de catalogage fondé sur un nouveau schéma de description. Ce dernier ne se limite pas à signaler la présence de tel ou tel titre éditorial dans la bibliothèque de Pierre le Grand, mais prend en compte les particularités des exemplaires (présence, absence ou ajout de gravures; ouvrages reliés ensemble, état de l'exemplaire, notes marginales, etc.). Essentiellement réalisé par Irina Hmelevskih, ce travail de catalogage est actuellement presque terminé. Sa parution prochaine est attendue en russe et en traduction française.

6 Par ailleurs, plusieurs autres catalogues plus ou moins directement liés à ce projet ont été réalisés par les conservateurs de la BAN, en premier lieu, celui des manuscrits en langues européennes, signé par Irina Lebedeva ${ }^{3}$. Il faut ajouter à cela la description publiée du corpus des ouvrages que Pierre hérita de la Chambre des apothicaires ${ }^{4}$ ou encore les catalogues des bibliothèques des personnes proches de Pierre le Grand, comme celle de Vinjus, travail réalisé par Elena Savel'eva ${ }^{5}$.

7 Ce projet a donné également lieu au premier dossier d'articles de réflexion, publié dans le numéro 47/3, Juillet-septembre 2006, des Cahiers du Monde russe. À la différence de ce premier dossier "généraliste ", en bonne partie consacré à l'histoire de la bibliothèque, celui que nous présentons ici est plus spécialisé et permet de tester notre source de manière plus concrète, en y découpant le « corpus romain », c'est-à-dire les livres, guides ou recueils de gravures, consacrés à la Rome ancienne et à ses antiquités, ainsi qu'à l'architecture de la Rome moderne. Ce découpage a permis de reposer la question du modèle romain dans la création de Saint-Pétersbourg, ainsi que celle, plus générale, des sources d'inspiration ancienne et moderne dans les stratégies civiles et militaires de Pierre le Grand.

8 Reposer ces questions au contact des ouvrages de la bibliothèque du tsar - qui, parfois, portent les traces de leur utilisation et, d'autres fois, sont évocateurs par le simple fait de leur accumulation - et le faire en collaboration avec les collègues non spécialistes de la culture russe permet d'échapper aux clichés véhiculés par l'historiographie nationale, reproduits ensuite dans les cercles de la slavistique occidentale.

9 Certes, les difficultés de travailler avec ce fonds aux contours capricieux sont nombreuses. Il suffit de penser, par exemple, à la nécessité de relier chaque exemplaire de la bibliothèque à la mention du titre correspondant dans les multiples catalogues et dans les archives, ou encore à celle d'interpréter les absences et les lacunes, étant donné que, d'une part, la bibliothèque n'a jamais été composée selon les règles de la bibliophilie et, d'autre part, qu'elle fut souvent déplacée et restructurée aussi bien durant la vie du tsar qu'après sa mort. 
10 Malgré ces difficultés, l'analyse des exemplaires de la bibliothèque pétrovienne - et tout particulièrement les indications qui concernent la réception des livres - permet aux auteurs des articles qui composent ce dossier d'esquisser nombre de questions et d'hypothèses nouvelles.

11 Quels étaient, par exemple, les plans de la Rome antique que Pierre le Grand avait devant les yeux au moment de la conception de sa ville? La nécessité de s'entourer d'un corpus des autorités sur la topographie de la Rome antique était-elle sentie comme primordiale par Pierre? En allant encore plus loin, pouvons-nous imaginer que l'idée même d'une grande variété de livres traitant du même sujet lui était étrangère et qu'il était satisfait s'il pouvait disposer d'au moins un livre par "question"? Devons-nous remettre en cause l'image du tsar amateur des antiquités? Et même si le tsar russe aimait les antiquités passionnément, avait-il la possibilité de satisfaire sa passion dans les conditions que pouvait lui offrir le marché européen de l'époque? Quant aux livres d'antiquaria, est-ce peu ou beaucoup de n'en avoir qu'une quinzaine? Faut-il interroger (et de quelle manière) l'absence des ouvrages concernant les médailles et les monnaies ? Ou la présence massive des éditions de De' Rossi parmi les livres du tsar?

Pierre le Grand aurait-il été un «mauvais » antiquaire, passionné surtout par la Rome moderne qu'il n'avait jamais vu, cette dernière lui parvenant via sa version " nordique ", vénitienne, d'abord, et hollandaise ensuite? Aurait-il été décidément moderne dans les questions des architectures militaires? Mais n'est-ce pas pour être moderne qu'il fallait à tout prix posséder les reliques des Anciens? Et en tout cas, ne le fallait-il pas, pour être Européen?

13 Ayant pour but la propagande en images, n'éprouvait-il pas besoin des antiquités qu'essentiellement dans le domaine de l'iconographie, cette dernière concernant surtout le culte des empereurs? Les éditions romaines sur les arcs et les colonnes de triomphe des Anciens ne servaient-elles pas essentiellement ses fins de propagande?

Enfin, la « romanité » de Saint-Pétersbourg n'aurait-elle pas été l'affaire des générations de commanditaires et d'architectes qui apparurent avec le règne de Catherine II ? Ou, malgré tout, la fascination exercée par les gravures romaines avait-elle eu une incidence dès la naissance du projet pétrovien?

15 Voici quelques-unes des questions posées ou reposées de manière nouvelle par les auteurs du présent dossier.

\section{NOTES}

1. B.A. Uspenskij, Ju.M. Lotman, Otzvuki koncepcii «Moskva - Tretij Rim» v ideologii Petra Pervogo [Échos de la conception «Moscou, troisième Rome » dans l'idéologie de Pierre le Grand], in B.A. Uspenskij, Izbrannye trudy [Morceaux choisis], t. 1, M.: Gnozis, 1994.

2. E.I. Bobrova, éd., Biblioteka Petra I: Ukazatel'-spravočnik (La bibliothèque de Pierre I ${ }^{\text {er }}$. Catalogue et index), L. : BAN, 1978. 
3. I.N. Lebedeva, Biblioteka Petra I: Opisanie rukopisnyh knig [La bibliothèque de Pierre Irr : catalogue des manuscrits], SPb., 2003.

4. E.A. Savel'eva, Katalog knig iz sobraniia Aptekarskogo prikaza [Catalogue des livres de la Chambre des apothicaires], SPb. : BAN, 2006.

5. E.A. Savel'eva, Knigi iz sobranija Andreja Andreevič Vinjusa [Catalogue de la collection d'A.A. Vinjus], SPb. : BAN, 2008. 